MATEC Web of Conferences 22,01050 (2015)

DOI: $10.1051 /$ matec conf/ 20152201050

(C) Owned by the authors, published by EDP Sciences, 2015

\title{
Fault Diagnosis of Power System Based on Improved Genetic Opti- mized BP-NN
}

\author{
Pu Yuan, Jianlin Mao, Fenghong Xiang, Lian Liu \& Maoxing Zhang \\ College of Information Engineering and Automation, Kunming University of Science and Technology, Kunming, \\ Yunnan, China
}

\begin{abstract}
BP neural network (Back-Propagation Neural Network, BP-NN) is one of the most widely neural network models and is applied to fault diagnosis of power system currently. BP neural network has good self-learning and adaptive ability and generalization ability, but the operation process is easy to fall into local minima. Genetic algorithm has global optimization features, and crossover is the most important operation of the Genetic Algorithm. In this paper, we can modify the crossover of traditional Genetic Algorithm, using improved genetic algorithm optimized BP neural network training initial weights and thresholds, to avoid the problem of BP neural network fall into local minima. The results of analysis by an example, the method can efficiently diagnose network fault location, and improve fault-tolerance and grid fault diagnosis effect.
\end{abstract}

Keywords: BP neural network; grid fault diagnosis; hidden layer; Genetic algorithm; fault-tolerance

\section{INTRODUCTION}

The faults of power transmission and distribution system bring a huge loss on power companies and users [1]. It is in the necessary condition that reduces the interruption time of the power and enhances the reliability of power supply when the malfunction of the grid happens. Fault diagnosis of grid has begun since 1940 s, and its main purpose is to quickly and accurately locate the fault element ${ }^{[2]}$. Due to certain restrictions of the previous conditions, traditional diagnostic methods rely on artificial diagnostic method which relies on experience to locate the position of grid failure, and its accuracy is rather poor, and the speed of diagnosis is relatively slow. With the development of diagnostic techniques, expert systems of artificial intelligence and genetic algorithms, artificial neural network, Bayesian networks, fuzzy sets ${ }^{[3]}$ as well as other methods were introduced to the power grid fault diagnosis. However, these methods have their limitations, for example, the fault-tolerance of expert system is poor, and it is difficult to give a correct diagnosis; the operation of genetic algorithm is more complex, the problem should first be encoded, and then genetic algorithm finds the optimal solution and decodes, and the genetic algorithm is easy to fall into the "premature" ${ }^{4]}$; neural networks are easy to fall into local minimum ${ }^{[5]}$, and so on.

This article based on the advantages and disadvantages of genetic algorithm, by modifying the crossover of Genetic Algorithm, and applied to BP neural network, optimizes the initial weights and thresholds of BP neural network. This method can solve the problem easily falling into local minima and improve fault-tolerance and the accuracy of grid fault diagnosis.

\section{PRINCIPLE OF BP NEURAL NETWORK AND ITS APPLICATION IN GRID FAULT DIAGNO- SIS}

\subsection{Principle of BP neural network}

BP (Back Propagation) neural network was proposed by scientist team of Rumelhart and McCelland in 1986. It is a multilayer feedforward networks of trained by the error back propagation algorithm, and it's learning rule is to use the steepest descent method. BP network can learn and store a lot of input-output modes without prior revealing mathematical equations that describe the modes.

Neural network is an operational model, composed by a large number of interconnected nodes (or "neurons", or "cells"). Each node represents a specific output function, called the activation function. Each connection between two nodes represents a weight value of the connection signal.

The basic principle of BP neural network model process information is the input signal through the middle nodes, and generates an output signal by non-linear transform. Each sample of network training includes input vectors and the expected output. By adjusting the weights and thresholds between the input nodes and hidden nodes, as well as the weights and thresholds between hidden nodes and output nodes, so that it reduces bias between the network output and the desired output value. The training can be stopped until determine the network parameters (weights and thresholds) of the minimum error.

The topology of BP neural network model, including the input layer, contains one or more hidden layers and output layers ${ }^{[6]}$. Figure 1 shows a typical three-layer neural network topology. 


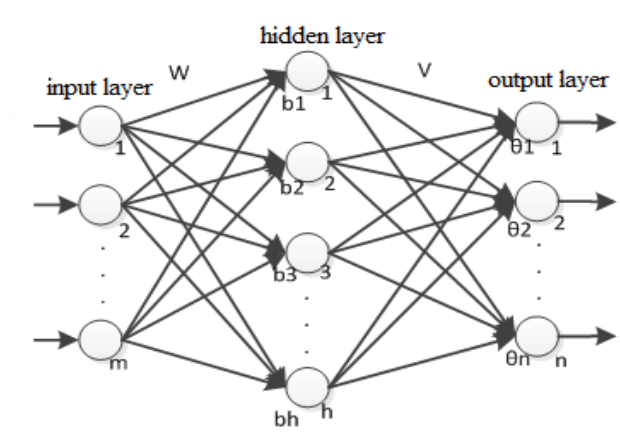

Figure 1. Three-level BP neural network topology

\subsection{Application of BP neural network in grid fault diagnosis}

It is still an unresolved problem to diagnose the grid malfunction quickly and accurately, and it is more difficult that the protection and circuit breaker refuse actions. In recent years, artificial neural networks have attracted the interest of researchers, because it has a good self-learning and adaptive ability and generalization ability. And the calculation of neurons in parallel will help to achieve real-time applications. In various models of neural networks, the most widely applied model is BP (Back-Propagation) neural network.

$\mathrm{BP}$ neural network realizes the mapping from input to output, and mathematical theory has been proven that it can implement any complex nonlinear mapping function, no model to understand its internal processes, simply entering to obtain output. Therefore, BP neural network is widely used in the grid fault diagnosis.

Literature [7-8] describe the specific application of BP neural network in the grid fault diagnosis: first, the action information of protection and circuit breaker is regarded as the input of network, and the fault area may occur as the output, constituting the fault decision table in order to establish a diagnostic model. Network trains fault decision table is divided into two parts, one is the forward propagation of signal, and another is the backward propagation of error. The former is input samples transmit to each input layer, and then transmit to the output layer. If there is an error between the output value and desired output value, then the error signal back propagation. Adjust the weights and thresholds, until the output value meets the requirement ${ }^{[9]}$.

BP neural network is a local optimization algorithm, and it may face a problem of local minima when the network trains fault decision table, resulting in the failure of training.

\section{IMPROVED GENETIC OPTIMIZATION BP-NN ALGORITHM DESIGN}

John Holland and other people in the United States at the university of Michigan, in order to simulate biological evolution of Darwin, designed a optimization algorithm in $1975^{[10]}$, called Genetic Algorithm (GA), but it was easy to fall into the "premature". This article describes improved Genetic Algorithm, in order to avoid genetic algorithm to fall into "premature", by modifying the cross of Genetic Algorithm. Then, use improved Genetic Algorithm in BP neural network, to find the optimal initial weights and thresholds. Finally, use training algorithm of BP neural network to find the optimal weights and thresholds in this local solution space.

\subsection{Improved genetic algorithm design}

Crossover is the most important operation of the Genetic Algorithm. We can obtain a new generation of individual by crossover. The new individual reflects the Characteristics of its Paternal. Crossover embodies the idea of information exchange. In this paper, we modified the crossover of traditional Genetic Algorithm. The way was changing the original crossover probability of traditional Genetic Algorithm, calculating a new crossover probability based on the original crossover probability. See the formula 1 below:

$p c 1=\frac{p c(f \max -f)}{(f \max -\text { faverage })}$

Where, $\mathrm{pc} 1=$ new crossover probability; $\mathrm{pc}=$ original crossover probability; fmax=maximum fitness value; $\mathrm{f}=$ current fitness value; and faverage $=$ average fitness value.

See specific improved Genetic Algorithm design below:

(1)Initialize the BP neural network, determine the network structure and learning rules, as well as the chromosome length of Genetic Algorithm (the chromosome length is the number of weights plus thresholds in hidden layer and output layer).

(2)Initialize the parameters of Genetic Algorithm (Including the number of iterations, population size, crossover probability and mutation probability etc.), as well as population and select the desired fitness function of Genetic Algorithm.

(3)Use the roulette wheel method to select several chromosomes to meet the requirements of the fitness function as a paternal of a new population.

(4)Deal with the male parent to produce a new generation of population by the changed crossover process and variation in the genetic algorithm.

(5)Repeat the steps 3 and 4, so that the chromosomes are constantly updated, until it reaches the target of training, and then find the best chromosome and decode for initial weights and thresholds of BP neural 
network.

For the above-described method, the key is to solve the coding scheme of genetic algorithm and select the fitness function. In this article, we use real-coded scheme in the Genetic Algorithm. The main purpose of improved genetic optimize BP neural network algorithm is to find the best chromosome, in order to make the minimum sum of squared errors of BP neural networks. Therefore, we can choose the sum of squared errors of BP neural networks as the fitness function of Genetic Algorithm. See the fitness function formula 2 below:

$$
f\left(x_{i}\right)=\frac{1}{2} \sum_{p=1}^{p} \sum_{j=1}^{m}\left(t_{j}^{p}-y_{j}^{p}\right)^{2}
$$

Where, tp $j=$ in the output layer of the $p$-th training sample, and the value of output corresponds to the J-th neuron output node; yp $\mathrm{j}=$ actual output value; $\mathrm{p}=$ the number of training samples; $m=$ the number of output layer neurons nodes; $x_{i}=$ the $i$-th chromosome in the population of Genetic Algorithm, $\mathrm{i}=1,2, \ldots, \mathrm{n}$, and $\mathrm{n}=$ the number of chromosomes in the population.

\subsection{BP neural network algorithm design}

(1)In this paper, by neural network architecture, we use three-layer structure, which contains a hidden layer, and this article chooses the number of hidden layer neurons which are the same with literature [1] The hidden layer activation function uses the S-type function, and the output layer activation function uses linear function, and the training function uses the L-M method.

(2)Use the improved genetic algorithm to search the weights and thresholds as the initial values of BP neural network, and then initialize the parameters of $\mathrm{BP}$ neural network (including the training times, the training goal, as well as the learning rate etc.).

(3)After completion of the above steps, network began training samples, and after completion of the training, we can call the network to enter the fault samples, and simulate fault samples by MATLAB.

\section{EXAMPLE DIAGNOSIS AND ANALYSIS}

\subsection{Diagnose process}

In this paper, we use the simple power distribution system of literature [1] as an example, and contrast the effects of improved Genetic optimize BP neural networks and traditional Genetic optimized BP neural networks by MATLAB simulate.

The simple power distribution system shown in Figure 2 has five fault areas $(\mathrm{Secl} \sim \mathrm{Sec} 5)$ and is equipped with over-current protections $(\mathrm{CO} 1 \sim \mathrm{CO} 5)$, as well as the circuit breaker protection switchs $(\mathrm{QF} 1 \sim \mathrm{QF} 5)$, Secl and Sec3 with the distance protection (RR1, RR3)

For power distribution model shown in Figure 2, select the fault decision table. Over-current protection switches and circuit breakers, as well as distance protections as the determine conditions of fault decision table, and as input in the neural network. Fault area (Secl-Sec5) as decision attribute in the fault decision table, and as output in the neural network. The fault decision table shown in Table 1 has 13 groups training samples. Each group has 12 determine conditions. Therefore, the input nodes of BP neural network are 12. The output of fault decision table has five fault areas and a trouble-free region. Therefore, the output nodes of BP neural network are 6.

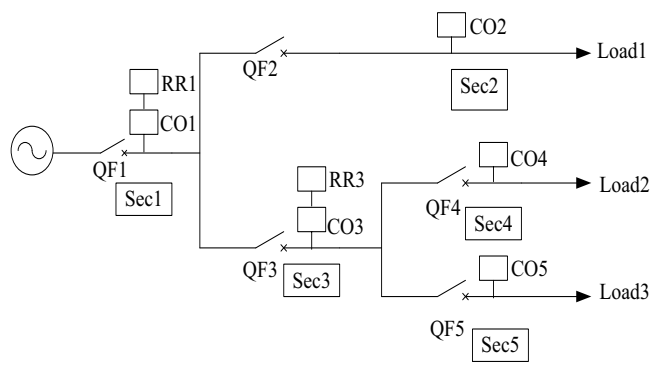

Figure 2. Simple power distribution system

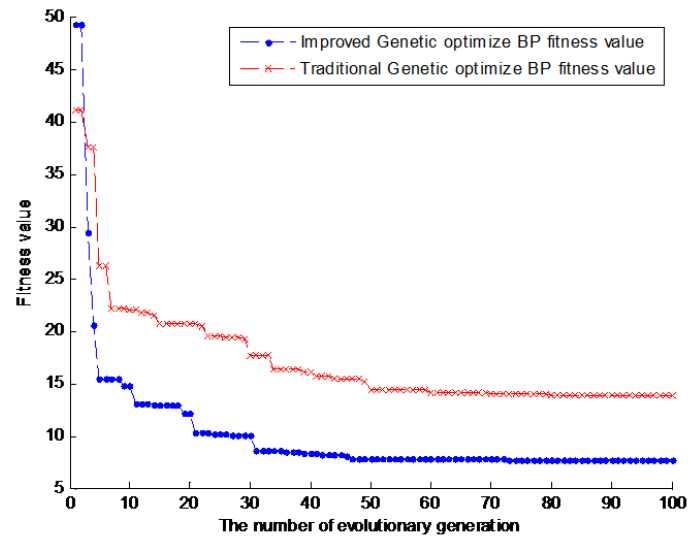

Figure 3. The optimal fitness value of two methods 
MATEC Web of Conferences

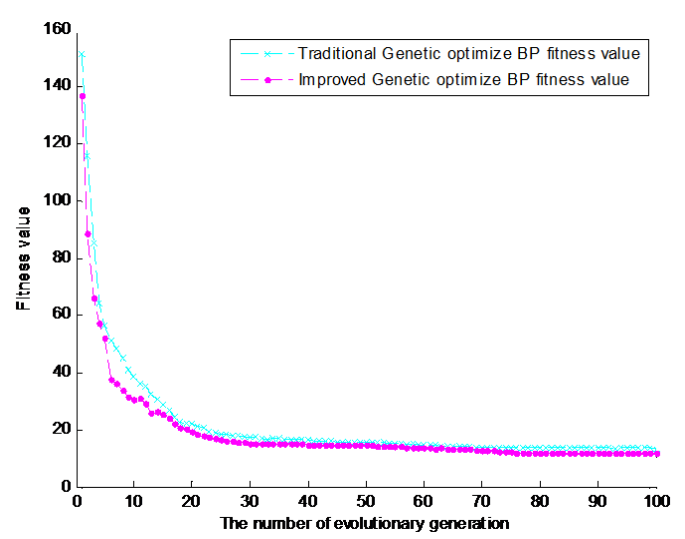

Figure 4. The average fitness value of two methods 
ICETA 2015

Table 1. Failure decision table

\begin{tabular}{|c|c|c|c|c|c|c|c|c|c|c|c|c|c|}
\hline No. & QF1 & QF2 & QF3 & QF4 & QF5 & $\mathrm{CO} 1$ & RR1 & $\mathrm{CO} 2$ & $\mathrm{CO} 3$ & RR3 & $\mathrm{CO} 4$ & $\mathrm{CO} 5$ & Fault area \\
\hline 1 & 1 & 0 & 0 & 0 & 0 & 1 & 0 & 0 & 0 & 0 & 0 & 0 & Sec1 \\
\hline 2 & 0 & 1 & 0 & 0 & 0 & 0 & 0 & 1 & 0 & 0 & 0 & 0 & $\operatorname{Sec} 2$ \\
\hline 3 & 0 & 0 & 1 & 0 & 0 & 0 & 0 & 0 & 1 & 0 & 0 & 0 & Sec3 \\
\hline 4 & 0 & 0 & 0 & 1 & 0 & 0 & 0 & 0 & 0 & 0 & 1 & 0 & Sec4 \\
\hline 7 & 0 & 0 & 1 & 0 & 0 & 0 & 0 & 0 & 0 & 1 & 0 & 1 & Sec5 \\
\hline 8 & 0 & 0 & 1 & 0 & 0 & 0 & 0 & 0 & 0 & 1 & 0 & 0 & Sec 4 or Sec 5 \\
\hline 9 & 1 & 0 & 0 & 0 & 0 & 0 & 1 & 1 & 0 & 0 & 0 & 0 & $\operatorname{Sec} 2$ \\
\hline 10 & 1 & 0 & 0 & 0 & 0 & 0 & 1 & 0 & 1 & 0 & 0 & 0 & Sec3 \\
\hline 11 & 1 & 0 & 0 & 0 & 0 & 0 & 1 & 0 & 0 & 0 & 1 & 0 & Sec4 \\
\hline
\end{tabular}

Note: 1 indicates a protective action, 0 indicates the protection is not activated.

\begin{tabular}{|c|c|c|c|c|c|c|c|c|}
\hline $\begin{array}{l}\text { Sam- } \\
\text { ple }\end{array}$ & $\begin{array}{c}\text { Diagnostic } \\
\text { method }\end{array}$ & Sec1 & Sec2 & Sec3 & Sec4 & Sec5 & None & $\begin{array}{c}\text { Training } \\
\text { result }\end{array}$ \\
\hline \multirow[t]{2}{*}{1} & GA-BP & 0.9999 & 0.0040 & -0.0043 & 0.0005 & 0.0024 & -0.0038 & Sec1 \\
\hline & I-GA-BP & 0.0178 & 0.9936 & -0.0064 & 0.0143 & -0.0015 & 0.0090 & $\operatorname{Sec} 2$ \\
\hline 2 & I-GA-BP & 0.0012 & 0.0017 & 1.0010 & 0.0058 & 0.0020 & 0.0118 & Sec3 \\
\hline \multirow[t]{2}{*}{3} & GA-BP & 0.0003 & 0.0006 & 1.0001 & -0.0004 & -0.0002 & -0.0011 & Sec3 \\
\hline & I-GA-BP & 0.0105 & 0.0082 & -0.0131 & 1.0101 & -0.0110 & -0.0057 & Sec4 \\
\hline \multirow[t]{2}{*}{4} & GA-BP & -0.0000 & 0.0001 & 0.0021 & 0.9997 & -0.0012 & 0.0002 & Sec 4 \\
\hline & I-GA-BP & 0.0081 & 0.0012 & -0.0034 & -0.0099 & 0.9938 & -0.0195 & Sec5 \\
\hline 6 & I-GA-BP & 0.0057 & 0.0111 & 0.0018 & 0.0074 & 1.0027 & 0.0013 & Sec5 \\
\hline \multirow[t]{2}{*}{7} & GA-BP & 0.0015 & 0.0036 & -0.0057 & 0.0009 & 1.0015 & 0.0040 & Sec5 \\
\hline & I-GA-BP & 0.0003 & 0.4781 & 0.1235 & 1.2310 & 0.9954 & 0.1142 & Sec or Sec 5 \\
\hline \multirow[t]{2}{*}{8} & GA-BP & 0.1112 & 0.3451 & -0.2587 & 0.9254 & 1.0235 & 0.2365 & Sec or Sec5 \\
\hline & I-GA-BP & 0.0338 & 0.9951 & 0.0055 & 0.0294 & 0.0016 & 0.0198 & $\operatorname{Sec} 2$ \\
\hline \multirow[t]{2}{*}{9} & GA-BP & -0.0004 & 1.0006 & 0.0000 & 0.0007 & -0.0001 & -0.0006 & Sec2 \\
\hline & I-GA-BP & 0.0347 & 0.0107 & 0.9939 & 0.0152 & 0.0044 & 0.0040 & Sec3 \\
\hline 10 & GA-BP & 0.0002 & 0.0004 & 1.0018 & -0.0005 & -0.0005 & -0.0010 & Sec3 \\
\hline
\end{tabular}

Note: In the diagnostic methods, I-GA-BP indicates improved Genetic optimize BP neural networks, GA-BP indicates traditional Genetic optimize BP neural networks. 
MATEC Web of Conferences

Table 3. Fault samples corresponding fault information

\begin{tabular}{|c|c|c|c|c|c|c|c|c|c|c|c|c|c|}
\hline No. & $\begin{array}{c}\text { QF1 } \\
\text { CO5 }\end{array}$ & QF2 & QF3 & QF4 & QF5 & $\mathrm{CO} 1$ & RR1 & & $\mathrm{CO} 2$ & $\mathrm{CO} 3$ & RR3 & $\mathrm{CO} 4$ & Fault area \\
\hline 1 & 1 & $1(0)$ & 0 & 0 & 0 & 1 & 0 & 0 & 0 & 0 & 0 & 0 & Sec1 \\
\hline 2 & 0 & 1 & 0 & 0 & 0 & 0 & 0 & 1 & 1 & $1(0) 0$ & 0 & 0 & Sec2 \\
\hline 3 & 0 & 0 & $0(1)$ & 0 & 0 & 0 & 0 & 0 & 1 & 0 & 0 & 0 & Sec3 \\
\hline 4 & 0 & 0 & 0 & $0(1)$ & 0 & 0 & 0 & 0 & 0 & 0 & 1 & 0 & Sec4 \\
\hline 5 & 0 & 0 & $0(1)$ & 0 & 0 & 0 & 0 & 0 & 0 & 1 & 0 & 1 & Sec5 \\
\hline 6 & 0 & 0 & $1(0)$ & 0 & 0 & 0 & 0 & 0 & 0 & 0 & 0 & 0 & None \\
\hline
\end{tabular}

Note: The data of outside the brackets are actual state, the data of inside the brackets are fault-free state.

Table 4. The diagnosis results of three algorithms

\begin{tabular}{|l|l|cccccc|c|}
\hline $\begin{array}{l}\text { Sam- } \\
\text { ple }\end{array}$ & $\begin{array}{l}\text { Diagnostic } \\
\text { method }\end{array}$ & Sec1 & Sec2 & Sec3 & Sec4 & Sec5 & $\begin{array}{l}\text { None } \\
\text { Diagnos- } \\
\text { tic result }\end{array}$ \\
\hline & I-GA-BP & 1.0028 & 0.0838 & -0.0353 & 0.1022 & 0.0082 & -0.4153 & Sec1 \\
1 & GA-BP & 0.8223 & 0.3173 & -0.0197 & -0.4777 & 0.2578 & -0.0637 & Sec1 \\
\hline & I-GA-BP & 0.2755 & 1.1619 & 0.7860 & 0.1367 & -0.4060 & -0.8956 & Sec2 \\
2 & GA-BP & 0.2017 & 0.7236 & 0.8587 & -0.2173 & 0.0134 & -0.3418 & indefinite \\
\hline & I-GA-BP & 0.0367 & -0.0238 & 0.8156 & -0.0168 & -0.1583 & 0.2978 & Sec3 \\
3 & GA-BP & 0.0544 & -0.2489 & 0.7880 & -0.3306 & 0.1028 & 0.4484 & Sec3 \\
\hline & I-GA-BP & 0.0474 & -0.0821 & 0.0909 & 0.8806 & -0.0222 & 0.3149 & Sec4 \\
4 & GA-BP & 0.0323 & -0.1037 & -0.2360 & 0.2922 & -0.7437 & 0.3811 & indefinite \\
\hline & I-GA-BP & 0.0966 & -0.0230 & -0.1899 & -0.0968 & 0.9676 & 0.3150 & Sec5 \\
5 & GA-BP & -0.1104 & -0.3811 & -0.2179 & 0.0483 & 0.8674 & 0.3836 & Sec5 \\
\hline & I-GA-BP & -0.1147 & -0.1502 & 0.2426 & 0.1319 & 0.0315 & 0.8366 & None \\
6 & GA-BP & 0.0311 & 0.3286 & 0.3335 & 0.1626 & 0.0608 & 0.5677 & None \\
\hline
\end{tabular}

Figure 3 is optimal fitness values of improved Genetic optimized BP neural networks and traditional Genetic optimize BP neural networks by MATLAB simulate. Figure 4 is average fitness values of improved Genetic optimize BP neural networks and traditional Genetic optimize BP neural networks by MATLAB simulate. We can see from two figures the best fitness and the average fitness tend to be stable after about 100 generations search.

In this article, the hidden-layer nodes choose 25 , and the network topology of two methods chooses a typical three-layer structure, and other parameters of two methods were the same. The training results of the two methods were shown in Table 2 .

Table 3 gives 6 fault samples of containing the protection device malfunction information. We know from the fault decision table, samples 1 and 6 are a circuit breaker malfunction, sample 2 is the over-current protection device malfunction, and samples from 3 to 5 refuse circuit breaker tripping. Fault samples diagnosis results are shown in Table 4.

\subsection{Analysis of experimental results}

In the same network structure and network parameters, contrasting the training effect of two methods, they all can accurately and effectively locate the fault location of training samples, and with little difference in the data, their training effects are obvious.

The diagnostic results of the fault samples containing the fault information analyzed that the effect of improved Genetic optimized BP neural networks is better than traditional Genetic optimized BP neural networks. The former can reach diagnostic results of $100 \%$, and the latter can reach diagnostic results of nearly $70 \%$. However, the fault diagnosis data of the latter are worse than the former.

\section{CONCLUSIONS}

We can see by comparing the simulation results of the two methods that the method of improved Genetic optimized BP neural networks can effectively locate the position of grid fault. The method of this article increases the fault-tolerance of grid fault diagnosis, and avoids the simple BP neural network to fall into local minimum easily. It is important for the combination of Genetic Algorithm and BP neural network, so that it can play their respective advantages, and improve the performance of grid fault diagnosis. The next work is to focus on the larger scale grid and more complex structures grid, and to check the effect of diagnosis and fault-tolerance.

\section{REFERENCES}

[1] Chao, Liu \& Zhengyou, He \& Jianwei, Yang. 2008. A Quantum Neural Network Based Fault Diagnosis Algonthm for Power Grid. Grid technology. 32(9): 56-60. 
[2] Xu, Zhang \& Juan, Wei \& Dongmei, Zhao. 2013. Research Course and Prospects of Power Grid Fault Diagnosis. Grid technology. 37(10): 2745-2753.

[3] Li, Bian \& Chenyuan, Bian. 2014. Review on intelligence fault diagnosis in power networks. Power Protection and Control System. 42(3): 146-153.

[4] Ding, S.F. \& Yu, J.Z. 2011. An optimizing BP neural network algorithm based on genetic algorithm. Artificial Intelligence Review. 36(2): 153-162.

[5] Ningsheng, Gong. 2011. AB network adjust the step and the hidden-layer neurons algorithm based on BP network. 13th IEEE Joint International Computer Science and Information Technology Conference (JICSIT 2011). Chongqing: IEEE.

[6] Yangming, Guo \& Congbao, Ran \& Xinyu, Ji. 2013. Analogous circuit fault diagnosis based on combinatorial optimization BP neural network. Northwestern Polytechnical University Journal. 31(1): 45-48.

[7] Jiaqiang, E. 2006. Intelligent Fault Diagnosis and Its Applications. Changsha: Hunan University Press.

[8] Chuangxin, Guo \& Chuanbo, Zhu \& Yijia, Cao. 2006. Research status and development trend of the power system fault diagnosis. Automation of Electric Power Systems. 30(8): 98-103

[9] Hong, Yan \& Yanping, Guan. 2009. Method to Determine the Quantity of Internal Nodes of Back Propagation Neural Networks and Its Demonstration. Control Engineering of China. 16(S1): 100-102.

[10] McCall, J. 2005. Genetic algorithms for modelling and optimisation. Journal of Computational and Applied Mathematics. 1(184): 205-222. 